\title{
Active Electromagnetic Damping for Lightweight Electric Vehicles.
}

\author{
Alista Fow \\ Department of Engineering, \\ University of Waikato, \\ Hamilton, New Zealand, \\ ajf8@students,waikato.ac.nz.
}

\author{
Mike Duke \\ Department of Engineering, \\ University of Waikato, \\ Hamilton, New Zealand \\ Dukemd@waikato.ac.nz
}

\begin{abstract}
Due to the low weight of most current electric vehicles, the effectiveness of commercial passive dampers is reduced when compared to current production automobiles. Active dampers allow for a significant reduction in the amount of road signal that affects the passenger body. However current active hydraulic dampers tend to be heavy and to have a power consumption that precludes their use in electric vehicles. By use of a linear electromagnetic element it is possible to create an active damper that has enough authority to provide active damping with a fraction of the weight and power consumption of a hydraulic system. A computer model of an active electromagnetic damper was constructed and the results were compared to a physical prototype. This verified the effectiveness of the damper and the low power consumption. This computer model was then scaled up for the simulation of a real world quarter car model. This simulation demonstrated that the use of the active linear electromagnetic damper was more effective at all frequencies when compared to an ideal passive damper. It was also demonstrated that the active electromagnetic damper had a similar mass to a passive damper and had a power consumption of more than an order of magnitude less than a comparable active hydraulic damper.
\end{abstract}

Keywords-component; Electromagnetic; damping; active; suspension.

\section{INTRODUCTION}

The use of a suspension system on an automobile has two main objectives: to reduce the accelerations felt by the passenger while traversing a roadway and to maximize the tyre contact with the road. In conventional suspension systems these two requirements can often be mutually exclusive. In practical suspensions a third major consideration is the travel distance of the wheel and the suspension elements. [1] The use of suspension elements in lightweight vehicles often exacerbates the situation with regards to comfort versus road handling.

The predominant suspension technology is the use of passive dampers and springs to isolate the passenger cabin.
While these decrease the passenger acceleration around the resonant frequency of the automobile/spring system, they increase the passenger accelerations at higher frequencies. This increase in passenger acceleration is greater for lightweight vehicles and has historically been a problem.

Modern technologies to overcome these limitations include the use of semi-active or active damper elements. A semiactive damper is usually based upon a passive damper that can be switched on or off by use of a controller. Most commercial semi-active dampers in vehicles use magnetorheological fluids that are subjected to magnetic fields generated by electromagnets. The most common control algorithms ae based upon Dean Karnopp's 'Skyhook' damper [2]. While this produces significant advantages over a purely passive damper, it also increases the system complexity and requires a power input which may affect the performance of an electric vehicle. As the semi-active damper uses a passive element to provide damping.

Over the last few decades there has been an interest in active suspensions systems. At least one hydraulic full active system has been made available on commercial automobiles and two such systems have been used in Formula One motor sport. The disadvantage of such a system is added weight, complexity and an increased power consumption. In a lightweight electric vehicle the advantages of a hydraulic active suspension are outweighed by these disadvantages.

An active suspension system requires the use of a controller to activate the forces generated. Just about all active damper systems use one of two control systems. These are the Skyhook damper proposed by Dean Karnopp in 1977 and Modal Control developed by Lotus [3].

It has been proposed that an active damper element could be constructed using electromagnets instead of hydraulic systems. This would reduce the potential weight and system complexity. Also, as an electric vehicle already has a robust electrical system, the addition of a power supply for an active damper would require little additional material.

The power consumption of a typical hydraulic active system is give as $3.8 \mathrm{~kW}$ by $\mathrm{Kim}$ and Okade [4]. For lightweight electric as proposed by [5] and [6], the energy demands for propulsion is in the range of $4.7-10.9 \mathrm{~kW}$. The use of an active hydraulic damper therefore increases the 
energy consumption of the vehicle by $35-81 \%$, along with a corresponding reduction in vehicle range. Using an slectromagnetic damper and spring system, Gysen, Paulides, Janssen and Lomonova, [7] researched a sedan being driven around the Nürbrgring and showed that while the peak power consumption of the vehicle was $2 \mathrm{~kW}$, the average power consumption for 'around town' driving was $16 \mathrm{~W}$. While the mass of the damper element is not revealed in this research, it can be seen that there is a significant difference in power consumption between an active hydraulic and electromagnetic suspension. For an innovate electric vehicle propulsion systems such as [8] and [9] where all four wheels are powered and can act as independent or centrally controlled units, a fully active suspension allows for the most effective and efficient use of this new technology

One of the simplest designs for an active electromagnetic damper, consists of an electromagnet travelling along the axis of the poles, through one or more coils. There is little research into whether such dampers would provide effective damping for lightweight electric vehicles. To be considered as effective in this application the damper should use a minimum of electrical power, have a light weight and reduce the passenger acceleration while increasing the force between the road and the tyre and it should be better at all of these elements than an equivalent commercial passive damper.

\section{A TheORETICAL ACTIVE DAMPER MODEL}

The simplest active damper consists of a single permanent magnet which is axially magnetised which travels along the axis through a cylindrical coil as shown in figure 1.

With a coil and magnet system, if a current is applied to the coil then a magnet field is generated in coil. The magnetic field of the coil then will interact with the magnetic field of the permanent magnet. This interaction produces a force.

The force generated on the coil, is derived from the Lorentz Force Law. For a permanent magnet and a single of

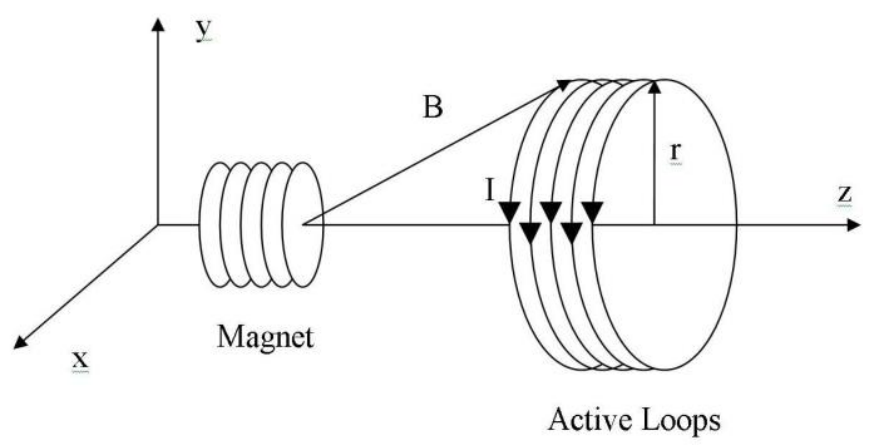

Figure 1 - A model of a magnet and coil. B is the magnetic field, $\mathrm{r}$ is the radius of the coil and I is the current in the coil. current carrying wire, the force on the wire is given by (1)

$$
F=B i L \sin \theta
$$

where $\mathrm{F}$ is the force in Newtons, $\mathrm{B}$ is the magnetic field in Tesla, $\mathrm{I}$ is the current in Amperes. $\mathrm{L}$ is the length of the wire in meters and $\theta$ is the angle between the loop and the $\mathrm{z}$ axis. As the loop is on the $\mathrm{x}-\mathrm{y}$ plane it is therefore normal to the $\mathrm{z}$ axis and the value of $\sin \theta$ is equal to one. As the loop and magnet are both centred on the $\mathrm{z}$ axis and by symmetry the value of $\mathrm{B}$ is constant around the loop, then the length of the wire is the circumference of the loop, so $L=2 r$ and the force equation becomes (2).

$$
F=2 \pi B I r
$$

It is well known that a permanent magnet can be modelled as a thin walled current carrying solenoid. For a solenoid with multiple coils as illustrated in figure 1, the magnetic field is $\mathrm{B}$ $\sin \_\theta=B_{z}$, the $z$ component of the field of the permanent magnet. The force generated by the coil is the sum of all of the loops of the solenoid and is given by (3)

$$
F=\sum_{i=0}^{i=n} 2 \pi r I B_{z}
$$

where $\mathrm{n}$ is the number of windings in the coil and $\mathrm{r}$ is the radius of the coil. While determination of the on-axis magnetic field for a thin walled solenoid is well known, determining the off-axis field is less well known. The method for determining this at any point in space using the thin walled solenoid model is derived from first principles in [10]. The magnetic field thus determined is then used in (3) to develop a lookup table of the forces for a cylindrical permanent magnet and coil using a unit current as described in [11].

The system is then modelled using the equation of motion of a single degree of freedom sprung system as given in (4)

$$
m \ddot{x}_{1}+c\left(\dot{x}_{1}-\dot{x}_{0}\right)+k\left(x_{1}-x_{0}\right)=0
$$

with $\mathrm{m}$ being the sprung mass, $\mathrm{c}$ being the dynamic damping coefficient, $\mathrm{k}$ being the spring constant, $\mathrm{x}_{0}$ being the position of the unsprung mass and $\mathrm{x}_{1}$ being the position of the sprung mass.

Two additional terms have to be added to (4) to yield the case of the active damper: $F_{\text {Active }}$ which is the force generated by the active damper and $F_{\text {Natural }}$ which is the natural damping forces provided by the damper when there is no active damping applied. The Natural damping force will have a combination of Coulombic and viscous damping. When added to (4) the result is (5) and gives the acceleration experienced by the sprung mass. 


$$
a=\frac{k\left(x_{0}-x_{1}\right)+F_{\text {Active }}+F_{\text {Natural }}}{m}
$$

The acceleration is then integrated to give the velocity of the mass. And integrated again to give the position.

The value of $\mathrm{F}_{\text {Active }}$ is obtained using the previously generated look up table for the magnet and the coil, inputting the distance between the magnet and the coil and then multiplying the result by the current supplied by the controller.

The controller chosen for this research is the full Skyhook damper proposed by Karnopp, Crosby and Harwood in [Karnopp 1974] and described by (6)

$$
F=-c \dot{x}
$$

where $\mathrm{F}$ is the force supplied by the damper, $\mathrm{c}$ is the damping coefficient that the active damper can supply and $\dot{x}$ is the velocity of the s prung mass relative to a fixed inertial point.

While there are many controller designs published, many of these strategies are to overcome shortcomings of the dampers they are used with. For research purposes the 'ideal' controller was chosen for use with the damper. The theoretical model of the damper and the controller is then modelled in VISSIM.

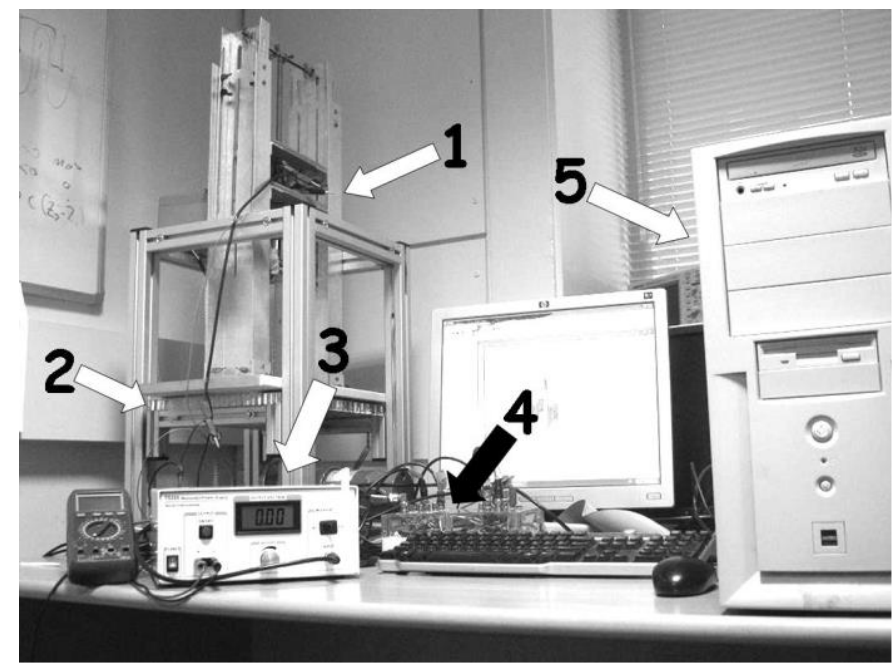

Figure 2 - The experimental apparatus for an active damper. (1) - the spring, damper, sprung mass and accelerometer, (2) The vibration surface. (3) The current amplifier. (4) The data input/output ports. (5) The computer with VISSIM REALTIME.

\section{A Practical Active Damper Model}

To validate the model a prototype damper was constructed as illustrated in figure 2 . The damper element was constructed using a coil of 120 turns in three layers of 40 windings each wound on to a PVC core. The mass was suspended from spring and had a natural frequency of $1.6 \mathrm{~Hz}$ for a1 $\mathrm{kg}$ mass. A Neodymium magnet with N35 rating and of physical dimensions $28 \mathrm{~mm}$ length and $19 \mathrm{~mm}$ diameter was attached to the sprung mass and this magnet produced a nominal magnetic field at the surface of the poles of $0.5733 \mathrm{~T}$.

The magnet was axially magnetised and assumed to have radial symmetry. The model of the magnet was used to generate a lookup table of the force generated by the damper. There was a better than $95 \%$ agreement between the predicted forces and the measured forces.

The Skyhook controller was constructed using VISSIM. REALTIME was used to input the information into the controller program and to output an analogue signal to an Accel Instruments TS200 Modulated Power Supply, which was used as a current amplifier to drive the active damper element. Through the use of a load resister, the power supplied one amp of current for each volt at the output from the controller.
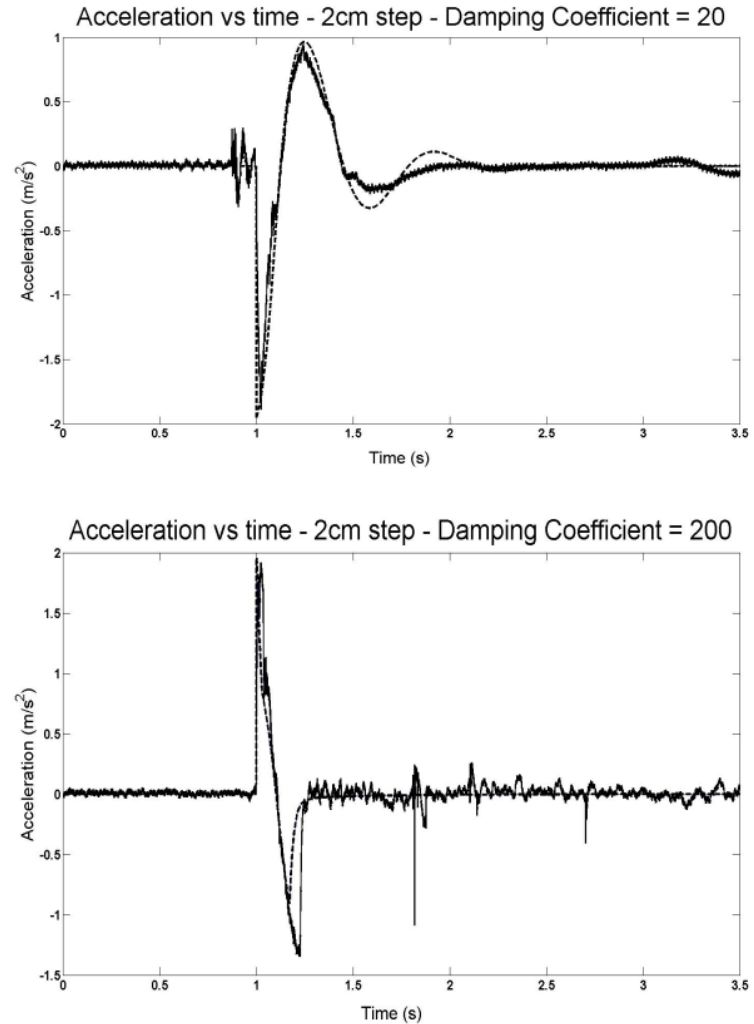

Figure 3 - In both figures the solid line are the measured values and the dashed line are modelled values. 
The signal input was supplied by an Analogue Devices ADXL325 accelerometer mounted upon the sprung mass. This was capable of measuring accelerations of $\pm 5 \mathrm{~g}$ in all three axis.

The data input/output was through a Measuring Computing PCI-DAS6014 data acquisition board which was installed in an IBM based computer. The PCI-DAS6104 was chosen because it also had analogue output capacity to drive the current amplifier.

The signal was processed to provide a self-levelling function for the data input to compensate for the offset in the accelerometer signal and the accelerometer drift. A custom integrator was used to supply the velocity signal in a timely manner.

\section{TESTING OF THE MODEL}

To determine the effectiveness of the damper model a series of experiments were conducted. In the time domain the model was tested with a damping coefficient of $20 \mathrm{~N} \mathrm{~s} / \mathrm{m}$ and $200 \mathrm{~N} \mathrm{~s} / \mathrm{m}$. The higher damping efficient provided an effective damping while the lower coefficient allowed a closer examination of the model. A step input of $2 \mathrm{~cm}$ was supplied to the sprung mass. There is a close relation between the predicted and measured values, as is illustrated in figure 3 ,

The damper was tested with a pseudo-sine wave input at a variety of frequencies from below the resonant frequency of the system to approximately $9 \mathrm{~Hz}$. The upper frequency limit was determined by the experimental apparatus. A series of experimental data runs were conducted over the frequency range and compared to the theoretical model. The magnet was the same $29 \mathrm{~mm}$ long neodymium magnet as used in previous experiments. The coil was the single coil as described previously. The damper value was set at $100 \mathrm{Ns} / \mathrm{m}$ and the time step for the VISSIM simulator was set at 0.001 seconds.

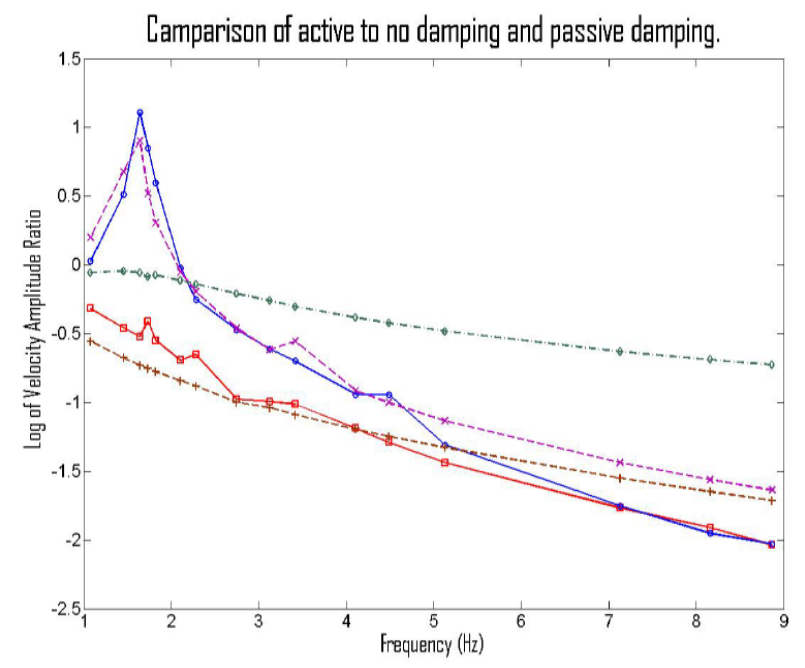

Figure 4. Active, Passive and No Damping -Frequency Domain. Red '_'s were active experimental. Brown '+'s were active modelled. Blue '_s were undamped experimental. Purple 'x's were undamped modelled. Green '_'s were ideal passive modelled.
A comparison was conducted between the measured nondamped motion of the system and the modelled non-damped motion of the system. The results are given in figure 4 . The velocity amplitude ratio is a standard measurement of damper performance and is determined by dividing the absolute velocity of the sprung mass by the absolute vertical velocity of the road surface.

It should be noted that as the motion of the mass was reduced then the effects of the accelerometer drift become more apparent and that the damper reacted to this drift. Also at the higher frequencies again the secondary motions become more apparent. There was a spike in the signal at the resonant frequency of the system. At frequencies above $5 \mathrm{~Hz}$ the results are more qualitative than quantitative.

Both the simulated and experimental active e.m. damper with Skyhook controller gives superior vibration performance compared to the passive damper at all frequencies. At lower frequencies the active damper was superior to the undamped suspension. At high frequencies the secondary effects had an effect on the performance of the active damper. However even with these secondary effects the performance of the active damper was similar to the undamped system. The average power consumption of the active damper was 0.105 Watts.

\section{SCALING UP FOR A PRACTICAL VEHICLE}

To determine the practicality of an electromagnetic damper in an electric vehicle the model of the was scaled and a two degree of freedom quarter car model was used. A smooth road surface model was also constructed using 12 different sine waves ranging from 0.5 to $20 \mathrm{~Hz}$ and a total vertical travel of approximately $2 \mathrm{~cm}$. This could be scaled to represent various roughnesses of the road surface.

The sprung mass was $50 \mathrm{~kg}$ and an unsprung mass of $50 \mathrm{~kg}$ was used. This value for the sprung mass represents the wheel, the suspension, an in hub motor and batteries associated with the wheel of an electric car.

The modelled damper was constructed of five Neodymium ND3522 magnets, each of $35 \mathrm{~mm}$ diameter and $22 \mathrm{~mm}$ length. The damper also consists of 6 coils each of 5 layers and each layer of 22 windings of $1 \mathrm{~mm}$ copper wire. The mass of the copper wire and the magnets is $0.985 \mathrm{~kg}$. With mountings and structural elements this is on the order of mass of a standard hydraulic damper.

TABLE 1 - THE PASSENGER ACCELERATION AND POWER CONSUMPTION FOR A DAMPER IN AN ELECTRIC CAR.

\begin{tabular}{|l|c|c|}
\hline Damper Type & R.m.s. acceleration $\left(\mathrm{m} / \mathrm{s}^{2}\right)$ & Power $(\mathrm{W})$ \\
\hline Passive & 0.579 & 0 \\
\hline Skyhook & 0.225 & N/A \\
\hline Active E.M. & 0.070 & 27.4 \\
\hline
\end{tabular}


The accelerations experienced by the passengers were modelled and the power consumption of the system was also modelled and are given in table 1 . When compared to a standard passive damper, the ideal semi-active reduced the acceleration by $61 \%$. The active e.m. damper reduced the acceleration by $88 \%$ compared to the passive damper and $69 \%$ when compared to the skyhook damper.

The average power consumption of $27.4 \mathrm{~W}$ per wheel produces a total power consumption of $110 \mathrm{~W}$. This represents $3 \%$ of the power consumption of a comparable hydraulic active suspension system on this surface.

\section{VI.CONCLUSION}

To be considered effective, the active e.m. damper was to use a minimum of electrical power, have a light weight and reduce the passenger acceleration while increasing the force between the road and the tyre. The damper should exceed the performance an equivalent commercial passive damper.

The modelled e.m. damper produces a good relationship between proposed and measured values. The active e.m. damper design proposed reduces the $88 \%$ when compared to a commercial passive damper. The power consumption is reduced by over an order of magnitude when compared to an equivalent active hydraulic damper.

\section{REFERENCES}

[1] Sharp, R. and S. A. Hassan, (1986), The relative performance capabilities of passive, active and semi-active car suspension systems, Proceedings Institution Mechanical Engineers, 200(D3), pp. 219-228.
[2] Karnopp, D., M. Crosby, and R. Harwood, (1974), Vibration control using semi-active force generators. Transactions of the ASME: Journal for Industry for Industry, 96(2), pp. 619-26.

[3] Williams, D. E. and W. M. Haddad., (1997), Active suspension control to improve vehicle ride and handling. Vehicle System Dynamics, 29, pp. $1-24$.

[4] Kim, S. and Okade, (2002), Variable resistance type energy regenerative damper using pulse width modulated step-up chopper. Journal of Vibration and Acoustics, 124, pp. 110-5.

[5] Duke, M., \& Anderson, T. N. (2008). The potential for battery electric vehicles in New Zealand.

[6] Duke, M., Andrews, D., \& Anderson, T. (2009). The feasibility of long range battery electric cars in New Zealand. Energy Policy, 37(9), 34553462 .

[7] Gysen, B., J. Paulides, J. Janssen, and E. Lomonova, (2008), Active electromagnetic suspension system for improved vehicle dynamics. In: IEEE Vehicle Power and Propulsion Conference. Harbin, China.

[8] Qiu, H., Liang, S., Qi, Z., \& Qin, H. (2012, November). A novel design of an in-situ steering for a 4-wheel independent steering in a 4-in-wheelmotor Drive Electric Vehicle. In Mechatronics and Machine Vision in Practice (M2VIP), 2012 19th International Conference (pp. 42-45). IEEE.

[9] He, P., Dong, Z., Liang, S., Qi, Z., \& Qiu, H. (2012, November). A novel design of all-wheel independent steering using regenerative inwheel motors for a four in-wheel-motor drive electric vehicle. In Mechatronics and Machine Vision in Practice (M2VIP), 2012 19th International Conference (pp. 51-55). IEEE.

[10] Kuns, K. (2007), Calculation of magnetic field inside plasma chamber. UCLA report, 2(3), pp. 1-11.

[11] Fow, A. (2014), Investigation into lowpower active electromagnetic damping for automotive applications, Unpublished doctoral dissertation, University of Waikato, Hamilton, New Zealand,. 